\title{
Hydrophone Arrays Assembled from PZT Ceramic Foams
}

\author{
H. KARA, ${ }^{a}$ R. RAMESH, ${ }^{a}$ R. STEVENS, ${ }^{a}$ N. JAYASUNDERE, ${ }^{b}$ \\ V. HUMPHREY, ${ }^{\mathrm{b}}$ and C. R. BOWEN ${ }^{\mathrm{a}}$ \\ ${ }^{a}$ Materials Research Centre, University of Bath, Bath, BA2 7AY, UK \\ ${ }^{\mathrm{b}}$ Department of Physics, University of Bath, Bath, BA2 7AY, UK
}

(Received August 8, 2003; in final form January 5, 2004)

\begin{abstract}
A $3 \times 3$ hydrophone array for low frequency applications was constructed from reticulated PZT ceramic foams. The hydrophone array was tested for receiving sensitivity and compared with single element PZT-air and dense PZT hydrophones. The composite array showed a flat response in the $20 \mathrm{kHz}-100 \mathrm{kHz}$ frequency range while the single element hydrophone displayed broadening radial resonance around $50 \mathrm{kHz}$. The flat, broadband response indicates that the radial resonance is suppressed by both porous nature of the PZT-air composites and the array construction of the hydrophone. The sensitivity of the array hydrophone was $-205 \mathrm{~dB}$ re $1 \mathrm{~V}_{\mu \mathrm{Pa}^{-1}}$.
\end{abstract}

Keywords: Piezocomposites; porous structures; hydrophones; underwater acoustics

\section{INTRODUCTION}

Piezocomposites with an active ceramic and passive polymer phase have found many applications as transducers. By introducing a passive polymer phase, radial resonances are suppressed which allows a flat, broadband response at low frequencies $(1 \mathrm{~Hz}-100 \mathrm{kHz})$. The composites can be configured by various methods [1], the most popular being a 1-3 configuration where the polymer decouples the lateral piezoelectric charge coefficients $\left(\mathrm{d}_{31}\right.$ and $d_{32}$ ) between individual ceramic pillars and maximise the contribution of the $\mathrm{d}_{33}$ axial charge coefficient [2].

These advantages can also be obtained using a 3-3 configuration of interpenetrating ceramic and polymer [3]. Porous PZT and PZT-polymer 3-3 piezocomposites have shown high hydrophone figures of merit (HFOM) compared with dense PZT $[3,4,6]$, with optimum HFOM values at $\sim 80 \%$ porosity [4]. This is achieved in a porous or cellular ceramic matrix impregnated with a polymer [4]. One advantage is that manufacturing of low cost 
cellular ceramics is well developed due to their use in molten metal filtering applications [5].

In this work, the construction of a hydrophone array based on cellular PZT ceramic foams is reported which enables high pore fractions to be obtained with high reproducibility. The array device was tested underwater and compared with single element hydrophones of the same material and conventional dense PZT ceramic.

\section{CERAMIC AND HYDROPHONE CONSTRUCTION}

The foam replication technique involves dipping polyethylene foam into a PZT-5H ceramic slurry and heat treating to remove the foam. The remaining ceramic skeleton is then sintered at $1200^{\circ} \mathrm{C}$ for 2 hours. Figure 1a shows examples of the structures manufactured from this technique. Ceramics were produced using a 20 ppi polyethylene foam which gave a ceramic volume fraction of $22.7 \%( \pm 1 \%)$. The ceramics sections of the array were attached to both sides of a $150 \mu \mathrm{m}$ thick copper electrode in series (i.e. same poling direction), as in Fig. 1b. Each row, composed of three pieces, was then attached to a small of copper electrode. A copper mesh was attached to electrodes for shielding and soldered to a $1.5 \mathrm{~m}$ cable of capacitance $42 \mathrm{pFm}^{-1}$. The assembly was finally moulded in polyurethane rubber for water isolation.

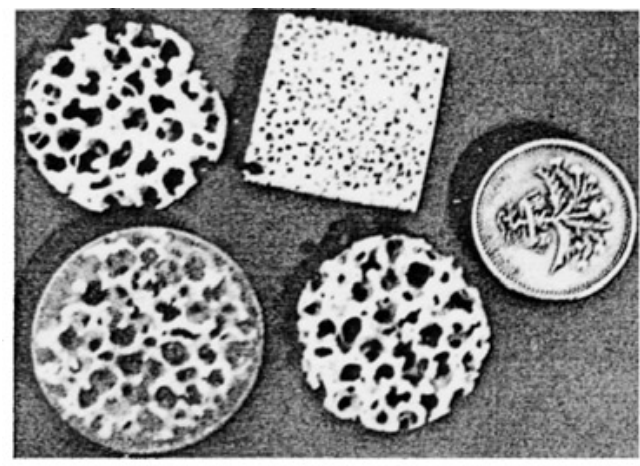

(a)

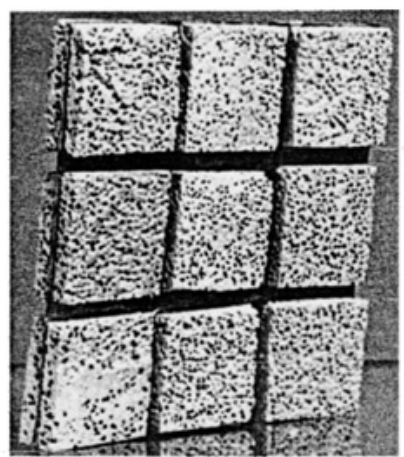

(b)

FIGURE 1 (a) Manufactured PZT-air and PZT-polymer piezocomposites from reticulated PZT ceramics. The square samples (PZT-air) were used in the hydrophone array in (b). 
The hydrophones were tested between $20-100 \mathrm{kHz}$ in a water tank using an impulse technique [7]. A truncated parametric array was used as the acoustic source to enable measurements over a wide frequency range without interference from surface/wall reflections. Hydrophone sensitivities were measured by comparing hydrophone outputs with a calibrated Brüel and Kjær 8103 hydrophone.

\section{RESULTS AND DISCUSSION}

Figure 2 shows that both the hydrostatic voltage constant $\left(\mathrm{g}_{\mathrm{h}}\right)$ and receive sensitivity of the 3-3 piezocomposite increase with increasing porosity/polymer content. The receiving sensitivity reaches an optimum between 90 and $80 \%$ porosity by volume [4], above which the material permittivity and device capacitance it too low for practical applications. Higher sensitivity values are obtained using polymer free composites (PZT-air) due to improved stress transfer into the active piezoelectric phase. The $22.7 \%$ ceramic volume PZTair composite was therefore chosen as the hydrophone material. Table I compares the properties of dense PZT-5H and the ceramic piezocomposite in the array, showing the composite has higher hydrostatic coefficients $\left(g_{h}\right.$ and $\left.d_{h}\right)$, lower permittivity and lower hydrophone capacitance.

The results of the acoustic measurements in the form of time-domain signals are shown in Fig. 3. The waveform for the piezocomposite device is sharp (Fig. 3a) but for a dense PZT hydrophone (Fig. 3b) is extended over a longer period of time. The short pulse duration is of use for highresolution applications, such as a hydrophone. Higher voltage outputs are also observed for the composite. Free field voltage sensitivities of the array
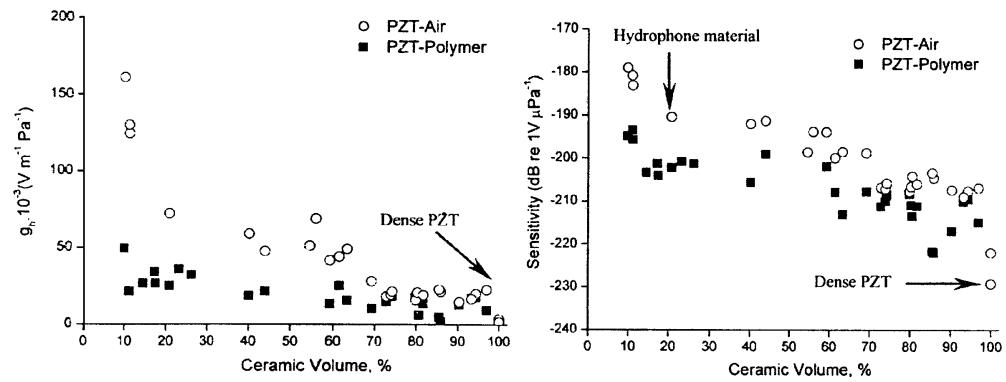

FIGURE 2 Composite (a) $g_{\mathrm{h}}$ and (b) calculated sensitivity increasing as a function of ceramic content. At low ceramic contents the permittivity and device capacitance is too low for practical applications. 


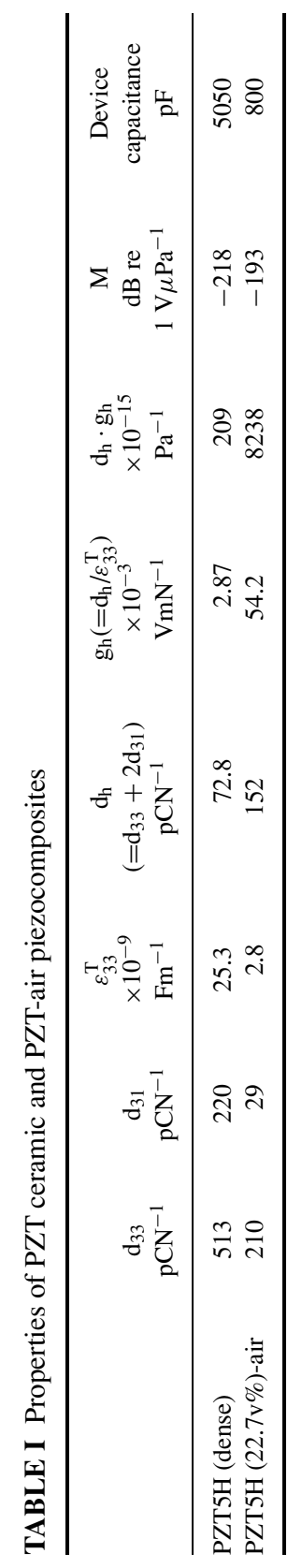




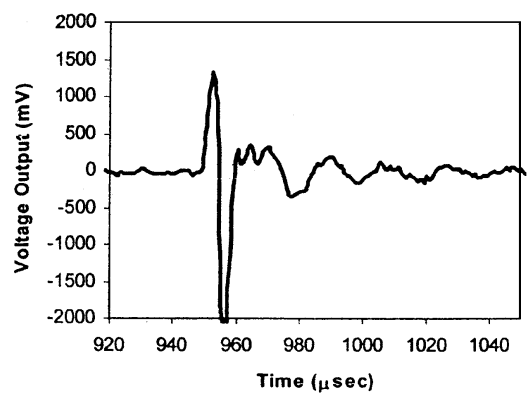

(a)

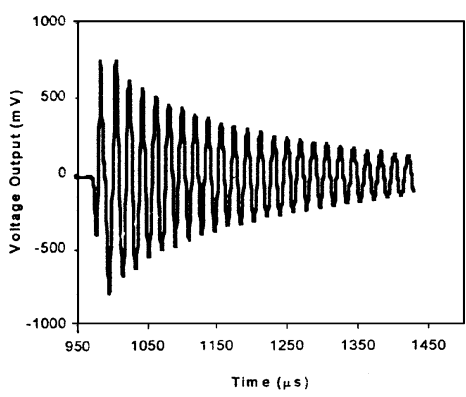

(b)

FIGURE 3 Time domain signals of (a) single element hydrophone (b) dense PZT hydrophone.

and single piezocomposite hydrophone are shown in Fig. 4a as a function of frequency. The sensitivities of the single element and array hydrophone are fairly constant over the frequency range studied. The dense PZT-5H shows sharp radial resonance in this frequency range (Fig. 4b). The array hydrophone showed the flattest response, while the PZT-air single element hydrophone displayed a broadening radial resonance at around $50 \mathrm{kHz}$. This indicates that the radial resonance is further suppressed by construction of an array. The sensitivity of the array hydrophone was $-205 \mathrm{~dB}$ re $1 \mathrm{~V} \mu \mathrm{Pa}^{-1}$. In summary, 3-3 composites show potential for high sensitivity, high resolution, broadband hydrophones. Further work will investigate the devices at lower frequencies, i.e. an omni-directional response.
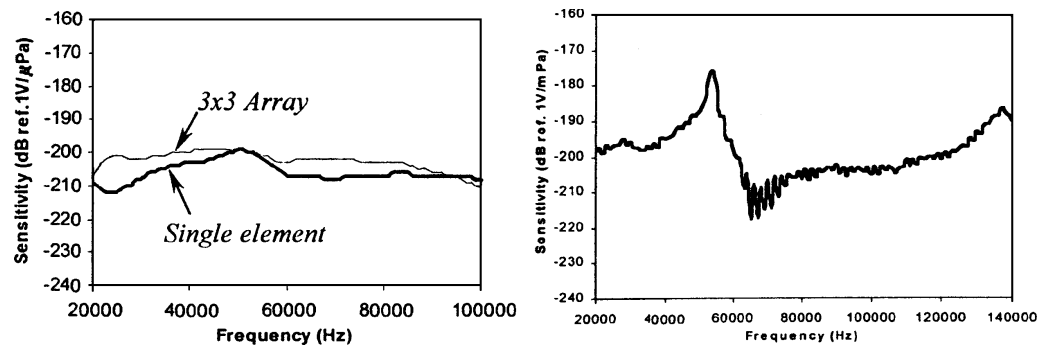

FIGURE 4 The receiving sensitivity of (a) single element and array hydrophones (b) dense PZT hydrophone. 


\section{ACKNOWLEDGEMENT}

The authors (HK) and (RR) are grateful to EPSRC and the Department of Science and Technology (India) for providing financial support, respectively.

\section{REFERENCES}

[1] R. E. Newnham, D. P. Skinner, and L. E. Cross, "Connectivity and piezoelectricpyroelectric composites," Mater. Res. Bull. 13, 525-536 (1978).

[2] K. A. Klicker, J. V. Biggers, and R. E. Newnham, "Composites of PZT and epoxy for hydrostatic transducer applications," J. Am. Ceram. Soc. 64, 5-9 (1981).

[3] M. J. Creedon, S. Gopalakrishnan, and W. A. Schulze, "3-3 composite hydrophones from distorted reticulated ceramics," Proceedings of the 9th IEEE International Symposium on Applications of Ferroelectrics 299-302 (1995).

[4] H. Kara, A. Perry, R. Stevens, and C. R. Bowen, "Interpenetrating PZT/polymer composites for hydrophones: models and experiments," Ferroelectrics 265, 317-332 (2002).

[5] S. Marselli, V. Pavia, C. Galassi, E. Roncari, F. Craciun, and G. Guidarelli, "Porous piezoelectric ceramic hydrophone," J. Acoust. Soc. Am. 106, 733-738 (1999).

[6] F. F. Lange and K. T. Miller, "Open-cell, low density ceramics fabricated from reticulated polymer substrates," Adv. Ceram. Mater.m 2, 827-831 (1987).

[7] R. J. Bubber, Underwater electroacoustic measurements, Naval Research Laboratory, Washington, USA (1970). 